\title{
APPROXIMATELY SUBHARMONIC FUNCTIONS
}

\author{
JOHN W. GREEN
}

1. Introduction. D. H. Hyers and S. M. Ulam [1] have introduced the notion of approximately convex function. A function $f$ is said to be $\epsilon$-approximately convex provided it satisfies the inequality

$$
f(\lambda x+(1-\lambda) y) \leqq \epsilon+\lambda f(x)+(1-\lambda) f(y),
$$

for $0 \leqq \lambda \leqq 1$. The positive number $\epsilon$ is fixed, and by regarding $x, y$ as vectors, we may apply the definition to any number of dimensions. For $\epsilon=0$, the definition reduces to that of convex function. Hyers and Ulam proved a theorem which amounts to the following: If $f$ is continuous and $\epsilon$-approximately convex in a convex domain in $n$ dimensions, there exists a convex function $g$ such that $g(x) \leqq f(x)$ $\leqq g(x)+k_{n} \epsilon$, where $k_{n}=1+(n-1)(n+2) / 2(n+1)$. In one dimension, since $k_{1}=1$, the most general continuous $\epsilon$-approximately convex function is obtained by adding to an arbitrary convex function a continuous function whose values lie between zero and $\epsilon$. In higher dimensions, since $k_{n}$ cannot be replaced by 1 , this decomposition is no longer possible.

Now in one dimension, the notions of convex function and subharmonic function are identical. Only a slight examination of the one-dimensional case is needed to suggest that approximately subharmonic functions may be a more natural extension to higher dimensions than approximately convex ones. Accordingly we define as follows:

Definition. $A$ function $f$ in a domain $D$ is $\epsilon$-subharmonic provided (a) it is upper semicontinuous, and (b) if $h$ is a harmonic function in a domain $D^{\prime}$ interior to $D$, which is continuous on the boundary of $D^{\prime}$ and dominates $f$ thereon, then in $D^{\prime}$.

$$
f(x) \leqq \epsilon+h(x) .
$$

This is not the only way in which one might naturally define $\epsilon$-subharmonic functions. One might take the definition as given, except to require that $D^{\prime}$ be a sphere, or one might take the analogue of the subaverage property and require only that $f(x)-\epsilon$ be dominated by the average of $f$ on a spherical surface centered at $x$. For $\epsilon=0$, these are all equivalent. For $\epsilon>0$, they are all different (even when $D$ is a sphere), although what relation exists between the three definitions

\footnotetext{
Presented to the Society, February 23, 1952; received by the editors February 7, 1952.
} 
is not obvious and affords an interesting problem. It is only with the strongest definition, namely the one given above, that one can prove the strongest theorem that is likely to come to mind; to wit:

Theorem 1. If $f$ is $\epsilon$-subharmonic in $D$, there exists a function $u$, subharmonic in $D$, such that

$$
u(x) \leqq f(x) \leqq \epsilon+u(x) .
$$

2. Greatest subharmonic minorants. To prove Theorem 2, we naturally would like to take for $u$ the greatest subharmonic minorant of $f$; that is, the greatest subharmonic function which does not exceed $f$, if such exists. The existence of a greatest subharmonic minorant of a given function is not a trivial matter to prove; in fact for general $f$, there is none. Its existence for continuous $f$, in two dimensions, was demonstrated by Sjöberg [2] in a proof depending heavily on conformal mapping. For upper semicontinuous functions, in any number of dimensions, its existence was proved by Brelot [3]. The latter's proof is involved with some of his work of a more profound nature on capacity, and depends in turn on certain results of H. Cartan and is not easy to trace back to its beginning; thus it may be of some interest to present an independent, although not essentially different proof. We first consider continuous $f$.

TheOREM 2. If $f$ is continuous in $D$ and admits a subharmonic minorant, it has a greatest subharmonic minorant, $u$. $u$ is continuous in $D$ and harmonic where it is less than $f$. If $D$ is a bounded Dirichlet domain and $f$ is continuous on $\bar{D}$, then $u$ is continuous on $\bar{D}$ and equals $f$ on the boundary.

Let $S$ be the class of functions subharmonic ${ }^{1}$ in $D$ and dominated by $f$. By hypothesis the class is not empty. For $x \in D$, we define

$$
u(x)=\sup _{s \in S} s(x) \text {. }
$$

We first show that $u$ is lower semicontinuous. Let $x_{0} \in D$, and $\eta>0$. There exists $s \in S$ such that $u\left(x_{0}\right)-\eta \leqq s\left(x_{0}\right) \leqq u\left(x_{0}\right) \leqq f\left(x_{0}\right)$. Then $s\left(x_{0}\right)-\eta<f\left(x_{0}\right)-\eta / 2$, and by the continuity of $f$ and the upper semicontinuity of $s, s(x)-\eta<f\left(x_{0}\right)-\eta / 2<f(x)$ for $x$ in some closed sphere $K$ about $x_{0}$. Let $\bar{s}$ be the function which agrees with $s$ outside $K$, and

${ }^{1}$ In the analogous treatment of the case of convex functions, one could use for $S$ either the class of convex functions or of linear functions dominated by $f$, and the result would be the same. However, in the present case, $S$ cannot be taken to be the class of harmonic functions dominated by $f$, the trouble being that a subharmonic function need have no harmonic support at a point. See [3, footnote 16]. 
is equal in $K$ to the harmonic function given by the Poisson integral with boundary values $s$. Then $s \leqq \bar{s}$ and $\bar{s}$ is subharmonic $[5, \S 5.4]$. In $K, \bar{s}(x)-\eta<f\left(x_{0}\right)-\eta / 2<f(x)$; also outside $K, \bar{s}-\eta<f$. Hence $\bar{s}-\eta \in S$ and $\bar{s}-\eta \leqq u$. We have

$$
\begin{aligned}
\lim _{x \rightarrow x_{0}} \inf u(x) & \geqq \liminf _{x \rightarrow x_{0}}(\bar{s}(x)-\eta)=\bar{s}\left(x_{0}\right)-\eta \\
& \geqq s\left(x_{0}\right)-\eta \geqq u\left(x_{0}\right)-2 \eta .
\end{aligned}
$$

Since $\eta$ is arbitrarily small, $\lim \inf u(x) \leqq u\left(x_{0}\right)$, and $u$ is lower semicontinuous and measurable.

Denote by $A(g, x, r)$ the average of the function $g$ over the surface of the sphere with center $x$ and radius $r$. It is understood that all spheres used will lie in $D$, together with their interiors. If $x \in D$ and $\eta>0$, there exists $s \in S$ such that

$$
u(x) \leqq \eta+s(x) \leqq \eta+A(s, x, r) \leqq \eta+A(u, x, r) .
$$

Here we have used the sub-average property of $s$. Since $\eta$ is arbitrarily small, it follows that $u(x) \leqq A(u, x, r)$. That is, $u$ has the sub-average property and is what Szpilrajn and Radó have called an almost subharmonic function. Szpilrajn [4] showed that there exists a subharmonic function $u^{*}(x)$ such that $u=u^{*}$ almost everywhere. For each $x$ in $D$, we have for almost all $r$,

$$
u^{*}(x) \leqq A\left(u^{*}, x, r\right)=A(u, x, r) \leqq A(f, x, r) .
$$

Since $f$ is continuous, $A(f, x, r) \rightarrow f(x)$ as $r \rightarrow 0$, and so $u^{*}(x) \leqq f(x)$. Thus $u^{*} \in S$ and $u^{*} \leqq u$. On the other hand, for almost all $r$,

$$
u(x) \leqq A(u, x, r)=A\left(u^{*}, x, r\right),
$$

and since $u^{*}$ is subharmonic, $A\left(u^{*}, x, r\right) \rightarrow u^{*}(x)$ as $r \rightarrow 0$. Thus $u=u^{*}$, and $u$ is subharmonic, upper semicontinuous, and hence continuous.

It is now clear that $u$ is harmonic whenever it is less than $f$. In fact, in a small sphere about such a point, let us replace $u$ by the harmonic function with the same boundary values to obtain a new member $\bar{u}$ of $S . \bar{u}$ cannot be less than $u$, since $u$ is subharmonic, nor more than $u$, since $u$ is maximal.

The remainder of the proof proceeds along standard lines, using a barrier.

Theorem 3. If $f$ is upper semicontinuous and dominates a subharmonic function, it has a greatest subharmonic minorant.

The proof is essentially the same as the proof below of Theorem 1, and will be omitted. 
In view of the remarks at the beginning of this section, it may be of some value to describe a function $f$ which has no greatest subharmonic minorant. In a space of $n$ dimensions, $n>2$, let $u_{k}, k=1,2, \cdots$, be the potential (based upon the function $1 / r^{n-2}$ ) of negative mass of constant density $-k^{2}$ spread on the sphere with center at 0 and radius $1 / k$. Then $u_{k}$ is subharmonic and negative. The total mass is $-\int_{0}^{1 / k} k^{2} r^{n-1} \Omega d r=\Omega / n k^{n-2}$, where $\Omega$ is the surface area of the unit sphere, and this mass tends to zero as $k \rightarrow \infty$. Now $u_{k}(0)$ $=-\int_{0}^{1 / k} k^{2} r^{n-1} \Omega d r / r^{n-2}=-\Omega / 2$. If $f$ is defined to be zero except at the origin, where it is equal to $-\Omega / 2$, then $f(x)=\lim _{k} u_{k}(x)=\sup _{k} u_{k}(x)$. Since $f$ is not subharmonic itself, it is clear that it has no greatest subharmonic minorant. $f$ satisfies the sub-average property, but has the wrong kind of semicontinuity.

3. Proof of Theorem 1. Since the $\epsilon$-subharmonic function $f$ is upper semicontinuous, it is the limit of a decreasing sequence of continuous functions, $\left\{f_{n}\right\}$. Let $E$ be a bounded Dirichlet domain interior to $D$, and let $u_{n}$ be the greatest subharmonic minorant of $f_{n}$ in $E$. The sequence $\left\{u_{n}\right\}$ is decreasing, and its limit $u$ is thus subharmonic. It is clear that it is the greatest subharmonic minorant of $f$ in $E$.

For each $x \in E$, either $u_{n}(x)=f_{n}(x)$ or $u_{n}(x)<f_{n}(x)$. In the latter case, $u_{n}$ is harmonic at $x$. Let $E^{\prime}$ be that component containing $x$ of the open set in $E$ where $u_{n}<f_{n}$. Then $u_{n}$ is harmonic in $E^{\prime}$ and equals $f_{n}$ on the boundary of $E^{\prime}$, by Theorem 2 and the continuity of the two functions $u_{n}$ and $f_{n}$. Since $f_{n}$ dominates $f$ on this boundary, $u_{n}+\epsilon$ dominates $f$ in $E^{\prime}$; that is, for every $x$ in $E, f(x) \leqq u_{n}(x)+\epsilon$. Allowing $n$ to become infinite, we have in $E$,

$$
u(x) \leqq f(x) \leqq u(x)+\epsilon .
$$

Theorem 1 is now proved, but for the subdomain $E$ of $D$. The remainder of the proof is made in an obvious fashion by using a nested sequence $\left\{E_{k}\right\}$ of bounded Dirichlet domains exhausting $D$. The corresponding sequence $\left\{u_{k}\right\}$ is decreasing and its limit is subharmonic. It is easy to see that this limit is the greatest subharmonic minorant of $f$ and satisfies the inequality (2).

It would be desirable to show that $f-u$ is upper semicontinuous, for then we could assert that the most general $\epsilon$-subharmonic function is obtained by adding to an arbitrary subharmonic function an arbitrary upper semicontinuous function whose values are between zero and $\epsilon$. We have not been able to prove or disprove this, however.

\section{REFERENCES}

1. D. H. Hyers and S. M. Ulam, Approximately convex functions, Proceedings of 
the American Mathematical Society vol. 3 (1952) pp. 821-828.

2. Nils Sjöberg, Sur les minorantes sousharmoniques d'une fonction donnée, Proceedings of the Ninth Scandanavian Mathematical Congress, Helsingfors, 1938, pp. 309-319.

3. Marcel Brelot, Minorantes sous-harmoniques, extrémales et capacites, J. Math. Pures Appl. (9) vol. 24 (1945) pp. 1-32.

4. E. Szpilrajn, Remarques sur les fonctions sousharmoniques, Ann. of Math. vol. 34 (1933) pp. 588-594.

5. T. Rad6, Subharmonic functions, Ergebnisse der Mathematik und ihrer Grenzgebiete, Berlin, 1937.

University of California, Los ANgeles, AND INSTITUTE FOR ADVANCED STUDY

\section{LATTICE ORDERING ON BANACH SPACES ${ }^{1}$}

\section{J. HEIDER}

In all that follows the letter $B$ will denote a given real Banach space, the letter $X$ a bicompact Hausdorff space, and the symbol $C(X)$ the space of all real-valued continuous functions $c(x)$ on the space $X$. The space $C(X)$ may be considered, among other ways, as a real Banach space under the norm $\|c(x)\|=1$.u.b. $|c(x)|, x \in X$, and as a linear lattice under the partial ordering $c_{1}(x) \geqq c_{2}(x)$ for $c_{1}(x)$ $-c_{2}(x)$ non-negative on $X$.

A space $B$ equivalent to a $C(X)$ under a linear norm-preserving isomorphism is, by transfer of the natural order on $C(X)$, susceptive of a partial ordering under which $B$ becomes both equivalent and lattice-isomorphic to $C(X)$. Thus the question of the purely Banachspace characterization of $C(X)$ is identical with the question of the linear normed lattice characterization of $C(X)$ provided the necessary and sufficient conditions under which a given Banach space is susceptible to a linear lattice ordering together with the additional conditions of the linear lattice characterizations are expressible in terms meaningful in all Banach spaces. This principle, applied in reference to the Kakutani [3] ${ }^{2}$ linear normed lattice characterization of $C(X)$, is implicit in the Clarkson [2] Banach space characterization of $C(X)$. The purpose of this note is to present the methods

Received by the editors January 19, 1952.

1 This note constitutes part of a thesis submitted to the University of Michigan. The author is grateful to Professor S. B. Myers for his advice.

2 Numbers in brackets refer to the references at the end of the paper. 